\title{
Small Bowel Prolapsed through Perforated Uterus following Illegal Induced Abortion
}

\author{
Francis Moïse Dossou ${ }^{1 *}$, Pierre-Claver Hounkpè ${ }^{2}$, Josiane Angeline Tonato Bagnan ${ }^{3}$, Justin Dénakpo ${ }^{3}$ and Pascal Sohou ${ }^{1}$ \\ ${ }^{1}$ Clinique Universitaire de chirurgie viscérale, Centre National Hospitalier Universitaire Hubert K. MAGA, Cotonou, Bénin \\ ${ }^{2}$ Service polyvalent d'anesthésie réanimation, CNHU-HKM, Cotonou, Bénin \\ ${ }^{3}$ Service de maternité de l'Hôpital de la mère et de l'enfant (HOMEL), Cotonou, Bénin
}

\begin{abstract}
Two women were admitted to the emergency service with small bowel prolapsed through the vagina after a curettage for voluntary termination of pregnancy. After a short resuscitation, laparotomy was performed for ileal resection followed by ileo-ileal end to end anastomosis, extensive washing of the abdominal cavity, digital curettage of the uterine cavity, debridement and suturing of uterine perforation. This serious complication of induced abortion is a consequence of the persistence of illegal induced abortion in Sub Saharan Africa. Prevention of this situation could be best archived by better information on contraception, better health education programs and more generally by rising educational attainment of girls.
\end{abstract}

Keywords: Induced abortion; Uterine perforation; Small bowel prolapsed; Vaginal evisceration; Sub-Saharan Africa

\section{Introduction}

Abortions, whether spontaneous or induced, are often associated with intra uterine instrumental manipulations to ensure the emptiness of the uterine cavity. Complications can arise in the aftermath of these procedures, and lead to dramatic consequences. We report two cases of evisceration through the vagina occurred in the aftermath of uterine curettage.

\section{Case 1}

An 18-years-old woman, G1, P0, was admitted to the emergency service with small bowel prolapsed through the vagina. She had consulted a few hours earlier in a care center for the termination of a pregnancy about 8 week's gestation. The operation was performed under neuroleptanalgesia, but during recovery of the patient, she complained of pelvic pain associated with heavy vaginal bleeding and the presence of small bowel loops getting out from the vagina. She was transferred to the emergency services of our hospital.

At the entrance, there was an impaired general condition with a shock: BP $90 / 50 \mathrm{mmHg}$, threading pulse $120 / \mathrm{min}$, mucosal pallor. The temperature was normal, $37^{\circ} \mathrm{C}$. There were numerous small bowel loops in purple crotch (Figure 1). The uterine globe was painful and the abdominal cavity seemed empty. The vaginal examination confirmed the presence of small bowel loops in the endocervix. The diagnosis of shock by evisceration per vagina due to induced abortion was confirmed, hence the indication for surgery. Laboratory tests had confirmed anemia. A brief resuscitation was undertaken before exploratory laparotomy. At laparotomy, the abdominal cavity was emptied of the small bowel that had herniated into the uterine cavity through a hole created iatrogenically at the posterior wall of the uterus. This hole was enlarged to reinstate all of the small intestine into the abdominal cavity. Re-staining of the intestine was observed over much of its length but about $2 \mathrm{~m}$ of ileum remained lifeless.

Ileal resection followed by ileo-ileal end to end anastomosis were performed as well as extensive washing of the abdominal cavity with saline supplemented with an antiseptic (povidone iodine), digital curettage of the uterine cavity, debridement and suturing of uterine perforation. A triple antibiotic was given intravenously for 5 days (ampicillin, gentamicin, metronidazole) as well as topical antiseptics. The postoperative course was uneventful. The patient was discharged on the $10^{\text {th }}$ postoperative day under estrogen and progestin drug.

\section{Case 2}

A 30-years-old woman, G1, P0, was admitted to the emergency service for small bowel prolapsed through the vagina. The patient had been accessed in a health center on the same day for metrorrhagia. Pelvic ultrasound has diagnosed ovular debris in the uterine cavity inducing performing curettage for cleanliness. During this maneuver, the operator, non-specialist, had been surprised by the arrival to the vulva of small bowel loops. That motivated the transfer of the patient urgently to hospital.

At the entrance, there was a normal general state with BP $120 / 80 \mathrm{mmHg}$, a regular pulse at $88 / \mathrm{min}$, a temperature at $37^{\circ} 5$, and conjunctiva slightly colored. Inspection noted purple small bowel loops injured in several places and bleeding coming from the vulva (Figure 2). Palpation of the abdomen revealed a painful uterine globe and a generalized abdominal defense. The vaginal examination confirmed the presence of small bowel loops in the endocervix. The diagnosis of small bowel evisceration due to instrumental abortive maneuvers was posed, hence indicating a laparotomy. Per operatory exploration showed uterine perforation about $1 \mathrm{~cm}$ in diameter, with an intramural oblique route about $4 \mathrm{~cm}$ long leading to the uterine cavity, many purple small bowel loops around the perforation, some of which were stripped of mesentery and devitalized, slow continuous bleeding of the part of the mesentery detached from its bowel.

Ileal resection followed by ileo-ileal end to end anastomosis were performed as well as extensive washing of the abdominal cavity with saline supplemented with an antiseptic (povidone iodine), digital curettage of the uterine cavity, debridement and suturing of uterine perforation. A gastric continuous aspiration was performed for 48 hours, until the resumption of intestinal transit, associated with a dual antibiotic therapy for 5 days (ceftriaxone, metronidazole) combined

*Corresponding author: Francis Moïse Dossou, Clinique Universitaire de chirurgie viscérale, Centre National Hospitalier Universitaire Hubert K. MAGA Cotonou, Bénin, E-mail: dosfm@yahoo.fr

Received March 28, 2012; Accepted May 19, 2012; Published May 20, 2012

Citation: Dossou FM, Hounkpè PC, Tonato Bagnan JA, Dénakpo J, Sohou P (2012) Small Bowel Prolapsed through Perforated Uterus following Illegal Induced Abortion. Surgery Curr Res 2:118. doi:10.4172/2161-1076.1000118

Copyright: (c) 2012 Dossou FM, et al. This is an open-access article distributed under the terms of the Creative Commons Attribution License, which permits unrestricted use, distribution, and reproduction in any medium, provided the original author and source are credited. 


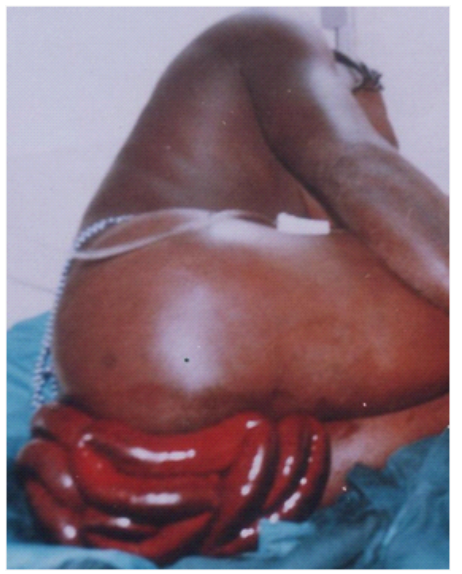

Figure 1: Small bowel loops prolapsed through the vagina.

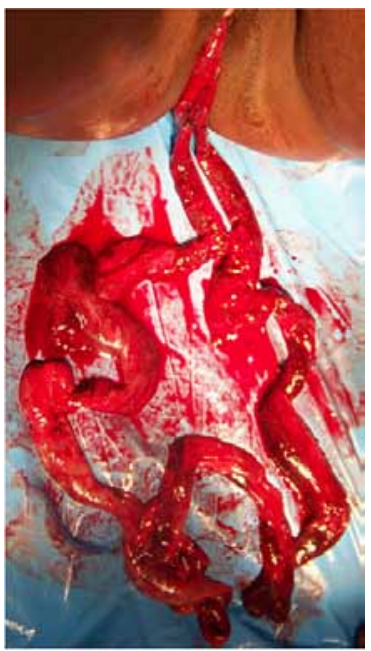

Figure 2: Small bowel loops coming from the vagina

with topical antiseptics. The postoperative course was uneventful and the patient was discharged on the $8^{\text {th }}$ postoperative day.

\section{Discussion}

Voluntary termination of pregnancy can be made medically or surgically. The surgical abortion uses instrumental endo-uterine maneuvers that should be performed by trained hands as they are purveyors of serious complications such as cervical lacerations and uterine perforations especially [1]. These complications are legion in Sub-Saharan Africa related to the persistence of illegal abortions $[2,3]$. Uterine perforation is usually source of peritonitis [4,5] but intestinal segment could inadvertently leave the abdominal cavity through this perforation created by curette or aspiration. Evisceration following induced abortion is known like a rare complication but it has already been described by several authors over the years $[4,6-8]$. This evisceration is serious as it cannot be resolved without surgery surrounded by resuscitation. Indeed, resuscitation plays an essential role in this situation where the hemorrhagic and septic shock is associated with the psychological and emotional shock in a patient weakened by guilt upstream and downstream of clandestine abortion.

In our second case, curettage would rather have taken place in a therapeutic purpose. This means that the legitimacy of the act is not only related to the indication for surgery, but also to the qualification and the rigor of the operator. Note that the medicalization of abortion has led to a decrease in complications and deaths in Western countries [1]. In black Africa, 5 to $10 \%$ of uterine perforations per abortion are always assigned to medical doctors $[9,10]$. Prevention of such complications requires more rigor and professionalism of doctors and also a decriminalization of abortion still illegal in many countries in Sub-Saharan Africa when performed without a medical reason. It could be best archived by a better information on contraception, better health education programs and more generally by rising educational attainment of girls $[8,11]$.

\section{Conclusion}

The vaginal evisceration due to abortion is a rare but very serious complication of abortion by intrauterine instrumental maneuver. It poses several problems, the most urgent task is to save the lives of women in treating hemorrhagic shock on the one hand, the intestinal damage and breach uterine other.

\section{References}

1. ANAES (2001) MANAGEMENT OF INTERRUPTION VOLUNTARY PREGNANCY UP TO 14 WEEKS. Service recommendations and professional references.

2. Nayama M, Gallais A, Wage H, Garba M, Idi N, et al. (2009) Complications of illegal abortion in a reference maternity of Niger: retrospective study of 151 cases. Black Afr Med 56: 171-175.

3. N'Guessan K, Doumbia Y, Effo N, Kakou C, Adjoby R, et al. (2007) Les perforations utérines au cours des avortements provoqués. Med Afr Noire 54 169-173.

4. Takongmo S, Nkwabong E, Pisoh-Tanggnyin C, Simeu C, Ndi Omgba R, et al (2010). Complications chirurgicales des avortements clandestins: A propos de 51 cas observés dans deux hôpitaux de Yaoundé. Clin Mother Child Health 7 : 1173-1177.

5. Buambo-Bamanga SF, Ekoundzola JR, Massengo R (2005) Complications chirurgicales des avortements provoqués clandestins au $\mathrm{CHU}$ de Brazzaville. Med Afr Noire 52: 139-144

6. Ntia IO, Ekele BA (2000) Bowel prolapse through perforated uterus following induced abortion. West Afr J Med 19: 209-211.

7. Oladapo OT, Coker AA (2005) Bowel prolapse and gangrene following vaginal vault perforation: an example of the menace of criminal abortion in Nigeria. Trop Doct 35: 177-178.

8. Ngowe NM, Ndjolo A, Bissou M, Ngonde SC, Ndom P, et al. (2006) The ilea evisceration per Vaginum: A serious post-abortion consequence was a case report. Med Afr Noire 53: 284-286.

9. Abauleth R, N'Guessan K, Kakou C, Konan KJ, Koffi A, et al. (2009) Prognosis of per abortum uterine perforations at the University Teaching Hospital of Cocody (Abidjan, Ivory Coast). Black Afr Med 56: 163-167.

10. Lebeau R, Guié P, Bohoussou E, Akpa-Bédi ES, Loukou Y, et al. (2012) An uncommon complication of unsafe induced abortion: Bowel prolapse through uterine perforation. Gynecol Obstet Fertil.

11. Akpadza K, Agba K, Tété V, Baéta S, Attignon A, et al. (2005) Connaissance et pratique de la contraception par les patients admises pour avortement provoqué clandestin à la clinique de gynécologie-obstétrique du CHU de LoméTokoin. Med Afr Noire 52: 345-351. 